\title{
Electrokinetic Evaluation of the Buffer Capacity of Soils
}

\author{
F. Hernández-Luis, ${ }^{a, *}$ D. Grandoso, ${ }^{a}$ M. Lemus, ${ }^{a}$ D. Benjumea, ${ }^{a}$ \\ C.D. Arbelo, ${ }^{b}$ D. Naranjo, ${ }^{c}$ M.V. Vázquez ${ }^{c}$ \\ ${ }^{a}$ Departamento de Química Física, Universidad de La Laguna, Tenerife, España \\ ${ }^{b}$ Departamento de Edafología y Geología, Universidad de La Laguna, Tenerife, España \\ ${ }^{c}$ Grupo de Electroquímica, Instituto de Química, Facultad de Ciencias Exactas y Naturales, \\ Universidad de Antioquia, AA. 1226, Medellín, Colombia
}

Received 15 September 2005; accepted in revised form 29 December 2005

\begin{abstract}
Based on the principles of the technique of electroremediation (electrochemical decontamination), experiments were carried out with the purpose to determine the buffer capacity of two soils from the region of Antioquia, Colombia, using a new method. These studies included the analysis of variation of the conductivity and residual $\mathrm{pH}$ in selected soils, after application of an electric field. From the different obtained parameters a function was defined combining the values of $\mathrm{pH}$ in zones close to the cathodic chamber with the corresponding one to the blank. This function allows obtaining a measurement of the buffer capacity of the soils. In this case the function represents the easiness for changing the $\mathrm{pH}$ when the electric field is applied and therefore it would not only have agricultural utility, but also to evaluate the behaviour in the case of a electrochemical decontamination treatment. The observed variations in the function are in agreement with those obtained when acid or bases solutions are added to soils.
\end{abstract}

Keywords: electrocharacterization, electroremediation, soils, $\mathrm{pH}$, conductivity.

\section{Introducción}

La determinación de parámetros fisicoquímicos de interés agrícola, suele formar parte de los análisis rutinarios en los laboratorios de suelos. Una de estas determinaciones sencillas, pero sumamente importante, es la determinación del $\mathrm{pH}$ del suelo y, relacionado con esto, la capacidad reguladora del mismo.

Aunque el valor de la acidez que presenta un suelo dependerá de una serie de factores que actúan conjuntamente, representa una magnitud de gran importancia

\footnotetext{
* Corresponding author. E-mail address: ffhelu@ull.es.
} 
puesto que controla el intercambio iónico, los procesos de disolución/precipitación, reducción/oxidación, adsorción y reacciones de complejación.

El pH del suelo está regulado, principalmente, por los componentes de la fase sólida del suelo: minerales arcillosos, monómeros y polímeros de hidroxialuminio, materia orgánica, etc. Este $\mathrm{pH}$ se modifica cuando se añaden fertilizantes o calizas, por ejemplo, las cuales en particular se emplean de rutina para corregir la acidez del suelo. Por este motivo la determinación del $\mathrm{pH}$, resulta importante es cuando se desea calcular el requerimiento de cal del suelo. En su determinación se emplean varios métodos que tienen en común la correlación del $\mathrm{pH}$ del suelo, con las dosis de enmiendas correctoras de composiciones definidas [1].

Para la determinación de la capacidad reguladora de un suelo suelen emplearse métodos que implican el contacto los suelos estudiados con distintas cantidades de $\mathrm{CaCO}_{3}$, incubándolos luego durante tiempos definidos a temperatura ambiente, al cabo del cual se determina el $\mathrm{pH}$ resultante [2,3].

Otro parámetro de interés agrícola, es el contenido salino ya que el nivel de sales solubles en el suelo y en su disolución afectan significativamente el crecimiento de las plantas. Debe considerarse que, además del contenido salino natural del suelo, puede producirse un incremento por riegos con aguas salinas o por el empleo intenso de fertilizantes. Para evaluar dicho incremento se usa como parámetro de medida la conductividad eléctrica, determinada en la disolución resultante después de someter los suelos a extracción con $\mathrm{H}_{2} \mathrm{O}$. Habitualmente, estos métodos implican el uso de relaciones de volumen $1: 2$ y $1: 1$ de suelo: $\mathrm{H}_{2} \mathrm{O}$ [1].

En el estudio de retención de nutrientes se requiere realizar una amplia variedad de análisis que demandan gran cantidad de tiempo de estudio y recursos. Así, por ejemplo, en los estudios de retención de fósforo se necesita analizar el contenido de materia orgánica, $\mathrm{pH}$ del suelo en distintas condiciones, capacidad de intercambio catiónico y bases intercambiables, $\mathrm{Ca}$ y $\mathrm{Mg}$ por absorción atómica, $\mathrm{K}$ y Na por fotometría de llama, retención de agua, contenido de arcilla y arena, y contenido de Al, Fe y P después de etapas de extracción [4].

Para realizar estudios como los mencionados anteriormente o estudios, por ejemplo, de dinámica de iones en suelos agrícolas, pueden ser necesarios hasta 60 días de incubación [5].

Con base en lo anterior, resulta claro que la posibilidad de contar con métodos analíticos que permitan de manera simple y rápida obtener alguno de los parámetros mencionados, constituye un importante e interesante desafío para los laboratorios de suelos.

En este sentido, considerando los fundamentos de la técnica de electroremediación de suelos, se presenta ésta como una posibilidad real para ser adaptada para la caracterización de los mismos (electro-caracterización). La técnica de electro-remediación implica la aplicación de un campo eléctrico continuo entre dos electrodos enterrados en el suelo, durante un cierto tiempo [617]. Con la aplicación de este campo eléctrico se consigue, dependiendo de diversos factores como el grado de humedad del terreno, la acidez del suelo, 
contenido de materia orgánica, tipo de material arcilloso, etc., movilizar las especies cargadas hacia los electrodos correspondientes (ánodo o cátodo).

Un aspecto importante derivado de la aplicación del campo eléctrico en el tratamiento, es la electrólisis del solvente que ocurre en los electrodos y que genera la aparición de frentes ácido y básico.

$$
\begin{array}{ll}
2 \mathrm{H}_{2} \mathrm{O}-4 \mathrm{e}^{-} \rightarrow \mathrm{O}_{2}+4 \mathrm{H}^{+} & \text {(ánodo) } \\
4 \mathrm{H}_{2} \mathrm{O}+4 \mathrm{e}^{-} \rightarrow 2 \mathrm{H}_{2}+4 \mathrm{OH}^{-} & \text {(cátodo) }
\end{array}
$$

La posibilidad que estos frentes puedan movilizarse por el interior del suelo con mayor o menos facilidad dependerá de las características del mismo, fundamentalmente de su capacidad reguladora de $\mathrm{pH}$.

En estudios a nivel de laboratorio, es posible utilizar celdas empleadas por los autores del articulo para estudios de electro-remediación de suelos para obtener, en un tiempo relativamente corto, un conjunto de medidas experimentales, con las cuales que permiten la interpretación de los distintos procesos electrodinámicos en el interior del suelo que involucran especies iónicas de interés agrícola, cuando se someten a un campo eléctrico continuo. Estas determinaciones pueden ser: medidas de $\mathrm{pH}$, conductividad, concentración y distribución de especies residuales después del tratamiento electroquímico; así como realizar estos análisis en las disoluciones remanentes en las cámaras de los electrodos y complementar todo esto con el seguimiento de la variación de la corriente de electrólisis durante el tratamiento electroquímico [15-20].

Aunque el conjunto de variables enumerado se ha empleado al estudio de la dinámica de iones de metales pesados contaminantes del suelo [15-20], en el estudio que es objeto del artículo se usó para obtener parámetros que permitan realizar un estudio semejante a los análisis clásicos realizados en laboratorios de suelos, por ejemplo para la determinación de la capacidad reguladora.

En este trabajo se presentan algunos resultados preliminares obtenidos en la determinación electrocinética de la capacidad reguladora de dos suelos de la región de Antioquia (Colombia) .

Estos estudios se emplean actualmente, para analizar el comportamiento de un conjunto de suelos de origen volcánico de Tenerife (España), los cuales presentan características similares a los colombianos y se aspira a desarrollar correlaciones válidas para unos y otros.

\section{Parte experimental \\ Células}

Como célula para llevar a cabo la electrolisis se utilizó un diseño empleado en anteriores trabajos [15-20], que permite incorporar una mezcla de unos 200 gramos de suelo con $100 \mathrm{~mL}$ de agua desionizada. En la Fig. 1 se presenta un esquema de dicha celda, indicando las principales características de la misma.

Para llevar a cabo el estudio, se aplicó mediante una fuente de potencial HEALT, una diferencia de potencial continua de $12 \mathrm{~V}$ entre dos electrodos de grafito de 
$2.15 \mathrm{~cm}^{2}$ de área, separado entre si $20 \mathrm{~cm}$. Esto genera un campo eléctrico de 60 $\mathrm{V} \mathrm{m}^{-1}$ que se mantuvo constantemente aplicado durante 48 horas.

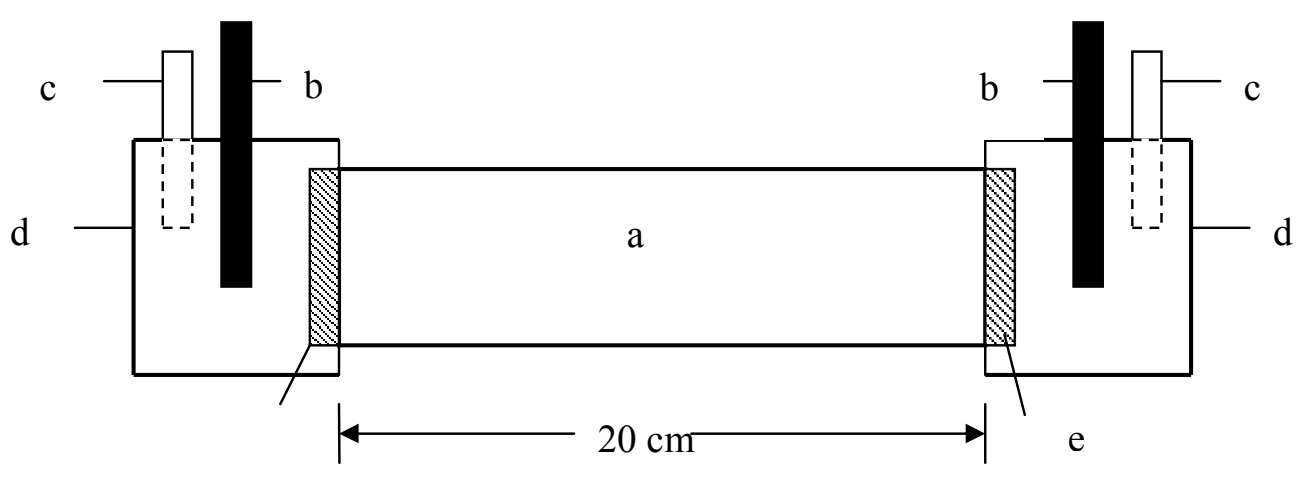
a- suelo-agua
b- electrodos de grafito
c- salida de gases
d- ventana transparente
e- rejilla perforada y papel de filtro

Figura 1. Esquema de la célula de electro-caracterización.

\section{Suelo estudiado}

En el presente trabajo se utilizaron dos suelos de cultivo con distintas características estructurales: uno proveniente del oriente antioqueño, denominado Suelo $O$ y otro de la región del Urabá antioqueño que fue designado como Suelo I. En la Tabla 1 se presentan las principales características de cada uno de estos suelos.

La distribución de tamaño de partícula fue determinada por el método del hidrómetro y el porcentaje de elementos gruesos se cuantificó por tamizado.

La materia orgánica fue determinada por el método de Walkley y Black y en ambos casos se trabajó con Horizontes A.

Tabla 1. Principales características de los suelos estudiados

\begin{tabular}{lcc}
\hline Parámetro & $\begin{array}{c}\text { Suelo } O \\
\text { (Tipic Hapludand) }\end{array}$ & $\begin{array}{c}\text { Suelo I } \\
\text { (Tipic Tropofluvent) }\end{array}$ \\
\hline Arena /\% & 40 & 70 \\
Limo / \% & 40 & 16 \\
Arcilla / \% & 20 & 14 \\
Materia orgánica / \% & 18.1 & 1 \\
Capacidad de intercambio efectiva & & 16.4 \\
/meq.100 $\mathrm{g}^{-1}$ & 16.3 & 16.3 \\
\hline
\end{tabular}


La capacidad de cambio cationica efectiva (CCCE) se determinó de la suma de los cationes cambiables extraídos con acetato amónico $1 \mathrm{~N}$ a $\mathrm{pH}=7$ y el aluminio de cambio extraído con $\mathrm{KCl} 1 \mathrm{~N}$ [21].

\section{Procedimiento}

En la Fig. 2 se resumen el procedimiento seguido para llevar a cabo los experimentos de electro-caracterización.

\section{Instrumental}

Las medidas de conductividad se llevaron acabo con un equipo SCHOTT Handdylab LF1.

El pH se midió utilizando un pHmetro ORION SA720.

\section{Valoración ácido base}

Para evaluar la capacidad reguladora de los suelos se realizaron valoraciones $\mathrm{pH}$ métricas sobre suspensiones de suelo:agua 1:0.4 y utilizando disoluciones $0.1 \mathrm{M}$ de $\mathrm{NaOH}$ y $\mathrm{HCl}$ para modificar el $\mathrm{pH}$.

Todos los resultados presentados en este artículo corresponden al promedio de tres repeticiones para cada uno de los suelo estudiado, siendo el máximo error encontrado en las medidas de $\mathrm{pH}$ de \pm 0.3 unidades.

\section{Resultados}

\section{pH del suelo después del tratamiento de electro-caracterización}

En la Fig. 3 se muestran los resultados obtenidos al analizar los distintos fragmentos de los suelos estudiados, una vez finalizado el tratamiento de electrólisis. De acuerdo con las reacciones de formación de oxígeno e hidrógeno en el ánodo y cátodo, respectivamente, se espera que los segmentos más próximos a las cámaras de los electrodos se vean afectados por los frentes ácido y alcalino que ahí se generan.

Para efectuar una comparación más rigurosa se debe tener en cuenta los valores del $\mathrm{pH}$ correspondientes al blanco, es decir de muestras de suelo contenidas en una celda de electro-caracterización pero sin aplicación de campo eléctrico, y que fueron: 6.41 para el Suelo I y 6.22 para el Suelo $O$.

Los resultados evidencian que, para las condiciones experimentales utilizadas, el suelo I presenta una mayor resistencia al cambio de $\mathrm{pH}$ generado por el frente alcalino.

\section{Variación de la conductividad residual}

Cuando se mide la conductividad que presentan las suspensiones de los distintos fragmentos de suelo, después del tratamiento de electro-remediación, se obtienen los resultados mostrados en la Fig. 4.

Los correspondientes valores de los blancos obtenidos con cada muestra son: $0.06 \mathrm{mS} . \mathrm{cm}^{-1}$ para el Suelo I y $0.16 \mathrm{mS} . \mathrm{cm}^{-1}$ para el Suelo $O$. 


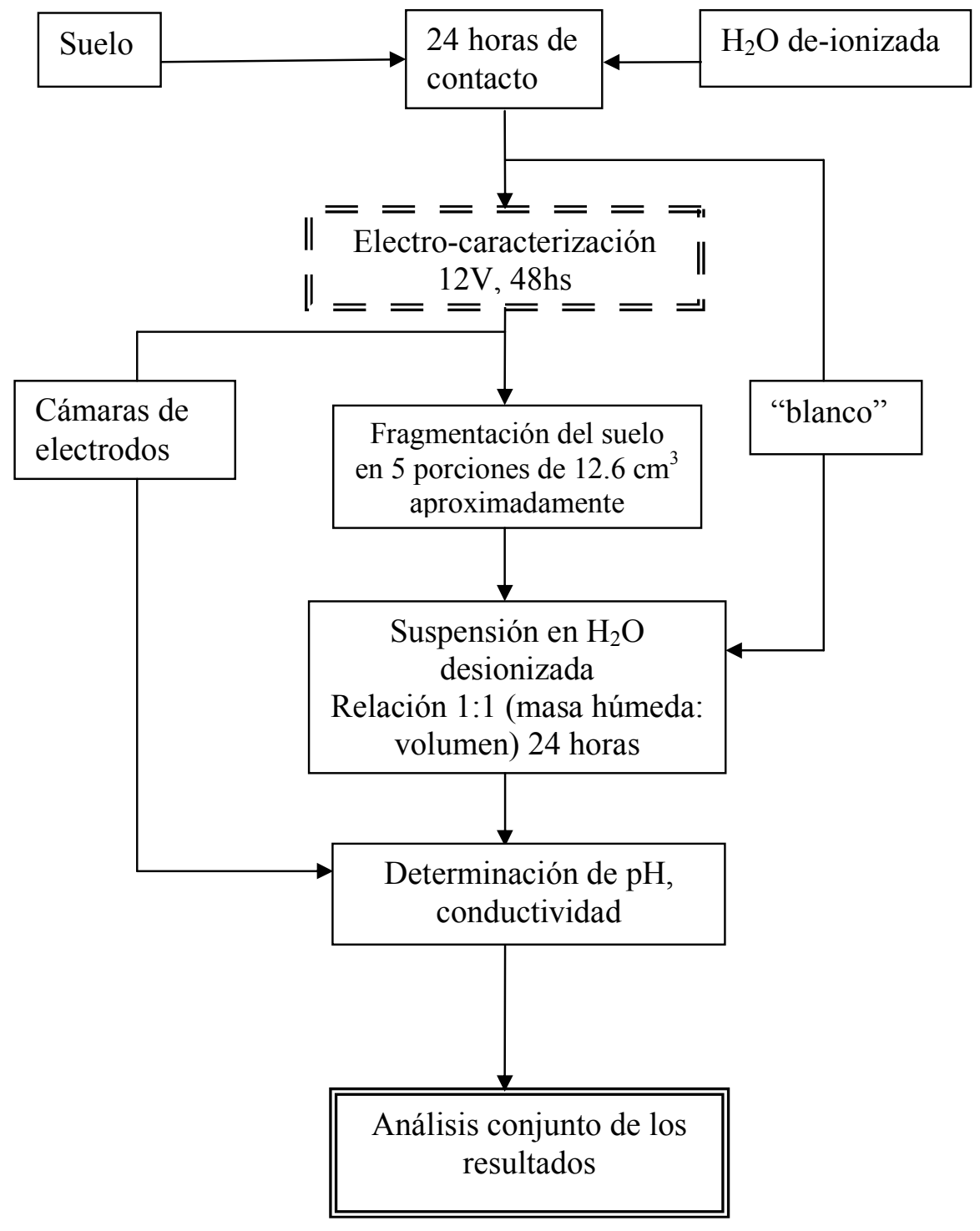

Figura 2. Resumen del procedimiento experimental. 


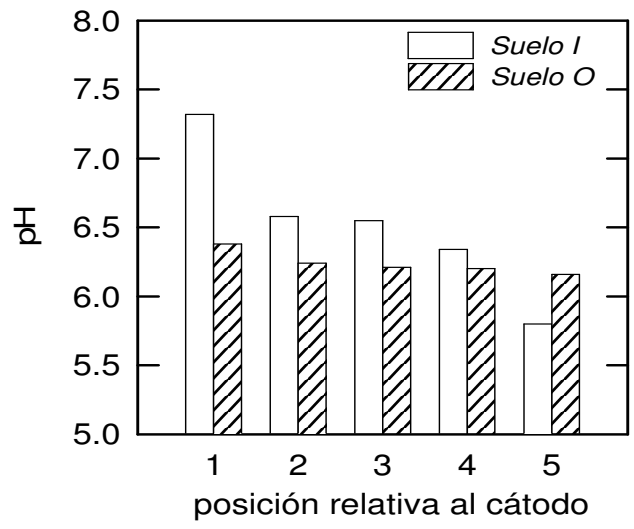

Figura 3. pH de los fragmentos de suelo después de la electrolisis.

Considerando que las condiciones experimentales fueron idénticas para ambos suelos, se observa que en el caso del Suelo $O$ se presenta una mayor concentración de iones en todos los segmentos comparados con el Suelo I. Además, en el primer suelo, se aprecia una tendencia que reflejaría un movimiento neto de iones hacia el cátodo, por acción del campo eléctrico aplicado. Esta variación, sin embargo, también puede ser interpretada con relación al aumento de $\mathrm{pH}$ que se produce en el segmento más próximo al cátodo y que conduciría a la formación de especies insolubles en esa zona.

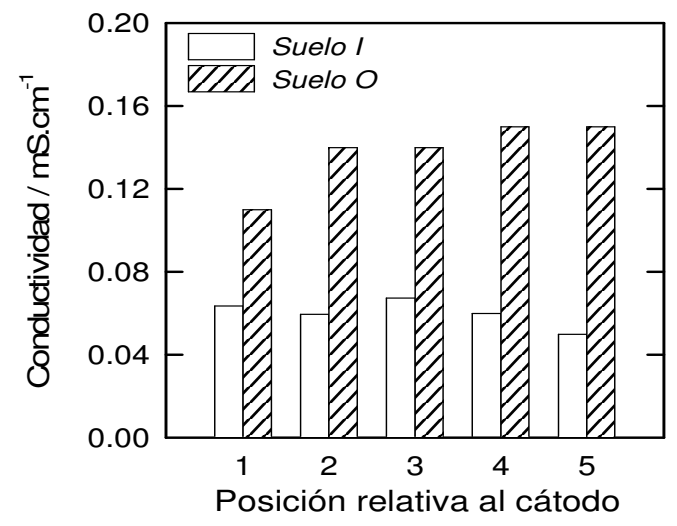

Figura 4. Conductividad de los fragmentos de suelo después de la electrolisis.

Considerando que las condiciones experimentales fueron idénticas para ambos suelos, se observa que en el caso del Suelo $O$ se presenta una mayor 
concentración de iones en todos los segmentos comparados con el Suelo I. Además, en el primer suelo, se aprecia una tendencia que reflejaría un movimiento neto de iones hacia el cátodo, por acción del campo eléctrico aplicado. Esta variación, sin embargo, también puede ser interpretada con relación al aumento de $\mathrm{pH}$ que se produce en el segmento más próximo al cátodo y que conduciría a la formación de especies insolubles en esa zona.

\section{Discusión y conclusiones}

Los resultados obtenidos con los dos suelos estudiados evidencian que es posible obtener una primera descripción de las características de los mismos, a través de un análisis relativamente rápido y sencillo.

Las medidas de conductividad reflejan un mayor contenido iónico en el Suelo $O$, que si bien, con los datos que se disponen, no es posible identificar, al menos se puede tener una idea del comportamiento de este material bajo la acción del campo eléctrico. Los valores de conductividad observados en las disoluciones remanentes en las cámaras de los electrodos después del tratamiento, se corresponden con la generación de los frentes ácido y alcalino como consecuencia de la electrólisis del medio acuoso.

Las variaciones de $\mathrm{pH}$ obtenidas pueden ser analizadas con mayor detalle con el propósito de interpretar el comportamiento de los suelos.

Como fue descrito en la Fig. 3, el aumento de pH observado en el segmento 1 (más próximo al cátodo) se corresponde con el contacto que tiene esta zona del suelo con el frente alcalino, generado en cercanías del cátodo. Por otra parte, la leve disminución del $\mathrm{pH}$ en el segmento 5 es consecuencia del otro frente generado.

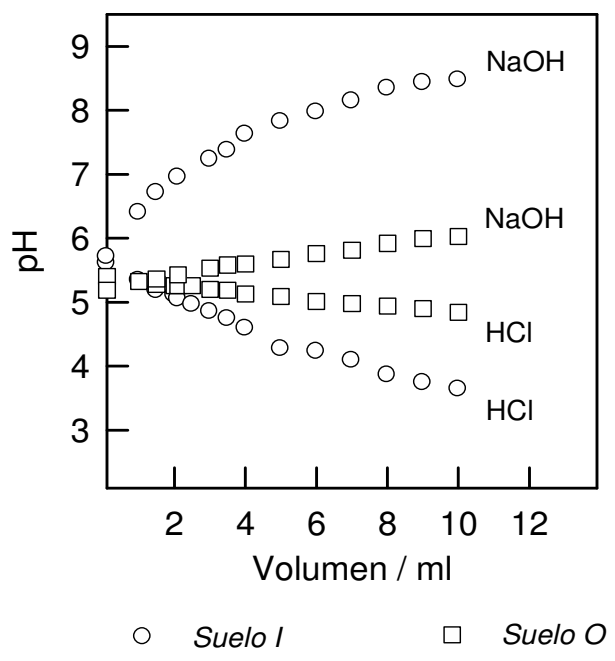

Figura 5. Variación del $\mathrm{pH}$ de las suspensiones de cada suelo tras añadir $\mathrm{NaOH} 0.1 \mathrm{M} \mathrm{o}$ $\mathrm{HCl} 0.1 \mathrm{M}$. 
El comportamiento de cada suelo frente al contacto con estas zonas de diferente $\mathrm{pH}$ está en relación directa con la capacidad reguladora que presenten los mismos.

Para verificar esto, se analizó la variación del pH en suspensiones de los suelos ante el agregado de disolución de $\mathrm{NaOH}$ y $\mathrm{HCl}$ respectivamente. La Fig. 5 muestra los resultados obtenidos. Se verifica una vez más que el suelo con mayor contenido en materia orgánica (Suelo O) presenta una mayor resistencia al cambio de $\mathrm{pH}$ cuando se añaden disoluciones ácidas y alcalinas.

Tabla 2. pH y conductividad de las cámaras electródicas y del blanco en cada suelo estudiado.

\begin{tabular}{ccccccc}
\hline & \multicolumn{3}{c}{ Suelo $O$} & & \multicolumn{3}{c}{ Suelo I } & \\
\cline { 2 - 7 } & $\begin{array}{c}\text { Cámara del } \\
\text { ánodo }\end{array}$ & $\begin{array}{c}\text { Cámara } \\
\text { del cátodo }\end{array}$ & Blanco & $\begin{array}{c}\text { Cámara } \\
\text { del ánodo }\end{array}$ & $\begin{array}{c}\text { Cámara } \\
\text { del cátodo }\end{array}$ & Blanco \\
\hline $\begin{array}{c}\mathrm{pH} \\
\begin{array}{c}\text { Conductividad } \\
\left(\mathrm{mS.cm}^{-1}\right)\end{array}\end{array}$ & 2.66 & 10.81 & 6.22 & 2.58 & 11.16 & 6.41 \\
\hline
\end{tabular}

Para intentar cuantificar esta propiedad se tuvo en cuenta los valores de $\mathrm{pH}$ obtenidos en los segmentos más próximos a las cámaras de los electrodos, así como los correspondientes a las disoluciones de las cámaras y a los blancos ya mencionados. En la Tabla 2 se reportan los valores anteriores así como los de las cámaras de los electrodos para el caso de los blancos. Este último caso se consideró para evaluar cuanto del comportamiento observado con el campo eléctrico se corresponde a procesos de difusión.

A partir de los valores mencionados se definió la función " $f$ " como sigue:

$$
f=1-\left(\frac{p H_{1}-p H_{b}}{p H_{-}-p H_{b}}\right)
$$

donde, $\mathrm{pH}_{1}$ es el $\mathrm{pH}$ del segmento más próximo a la cámara del cátodo, $\mathrm{pH}$ corresponde al $\mathrm{pH}$ de la disolución de la cámara del cátodo, $\mathrm{pH}_{\mathrm{b}}$ es el $\mathrm{pH}$ del suelo estudiado como blanco del experimento.

Del análisis de esta expresión matemática pueden obtenerse dos casos extremos:

a) que $\mathrm{pH}_{1}=\mathrm{pH}_{\mathrm{b}}$; en este caso la función toma valor unitario y sería equivalente a tener una capacidad reguladora máxima.

b) que $\mathrm{pH}_{1}=\mathrm{pH}_{\text {- }}$; en este caso la función toma un valor nulo y se correspondería a una capacidad reguladora cero.

Cuando se aplica esta ecuación a los resultados obtenidos con los suelos estudiados se obtiene una $f$ de 0.81 para el Suelo $I$ y una $f$ de 0.96 para el Suelo $O$, lo que es coherente con lo observado experimentalmente y que hemos discutido antes. 
De esta manera se verifica que es posible postular esta función como una manera de determinar la capacidad reguladora que presentan los suelos bajo la acción de un campo eléctrico. Esta herramienta puede ser de interés tanto desde el punto de vista de la caracterización con fines agrícolas, así como para analizar los efectos del tratamiento de descontaminación electroquímica ante una eventual contaminación.

En este primer estudio se trabajó con el segmento de suelo más próximo a la cámara del cátodo, ya que es la región donde se producen habitualmente los cambios más notorios de $\mathrm{pH}$ con relación al suelo. En caso de analizar el segmento más cercano al ánodo se obtiene un comportamiento similar al reportado más arriba, ahora con un $f$ de 0.84 para el Suelo I y un $f$ de 0.98 para el Suelo $O$.

\section{Agradecimientos}

Los autores desean agradecer a la Dirección General de Universidades e Investigación de la Consejería de Educación, Cultura y Deportes del Gobierno de Canarias por financiar parte de esta investigación a través del Proyecto Pi 2003/138.

Asimismo agradecen a la Agencia Española de Cooperación Internacional (AECI), dependiente del Ministerio de Asuntos Exteriores y Cooperación de España, por financiar las movilidades de algunos de los miembros del grupo de investigación, entre las Universidades de Antioquia (Medellín, Colombia) y La Laguna (Tenerife, España).

\section{Evaluación electrocinética de la capacidad reguladora de suelos}

\section{Resumen}

Basado en los principios de la técnica de electro-remediación (descontaminación electroquímica), se han llevado a cabo experimentos con la intención de determinar la capacidad buffer de dos suelos de la región de Antioquia (Colombia), utilizando un nuevo método. Estos estudios incluyen el análisis de la variación de la conductividad y $\mathrm{pH}$ residual en los suelos seleccionados, después de aplicar el campo eléctrico. A partir de los distintos parámetros obtenidos se definió una función que combina valores de $\mathrm{pH}$ en zonas próximas a la cámara del cátodo con el correspondiente al blanco. Esta función permite obtener una medida de la capacidad reguladora que muestran los suelos estudiados. En este caso se trata de la facilidad al cambio de $\mathrm{pH}$ cuando se aplica el campo eléctrico y por lo tanto no sólo tendría utilidad agrícola sino también para evaluar el comportamiento en caso de un tratamiento de descontaminación electroquímica. Las variaciones observadas en la función se corresponden con las obtenidas cuando se añaden disoluciones de ácido o base a los suelos.

Palabras claves: Electro-caracterización, electro-remediación, suelos, pH, conductividad. 


\section{Referencias}

1. Soil Analysis Handbook of Reference Methods, Council, Inc. CRC Press, Florida, 1999.

2. F.R. Magdoff, R.J. Bartlett, Soil Sci. Soc. Am. J. 49 (1985) 145-148.

3. R.L. Aitken, Aust. J. Soil Res. 30 (1990) 119-130.

4. C.E. Quintero, G.N. Boschetti, R.A. Benavidez, Commun. Soil. Sci. Plant. Anal. 30 (1999) 1449-1461.

5. G. Aguado-Lara, B. Etchevers, C. Hidalgo-Moreno, A. Galvis-Spínola, A. Aguirre-Gómez, Agrociencia 36 (2002) 11-21.

6. S. Chinthamreddy, K. Reddy, Journal of Soil Contamination 8 (1999) 197216.

7. M.M. Page, C.L. Page, Journal of Environmental Engineering 128 (2002) 208-219.

8. S. Pamukcu, A.Weeks, J. Wittle, Journal of Hazardous Materials 55 (1997) 305-318.

9. R.F. Probstein, R.E. Hicks, Science 260 (1993) 498-503.

10. K. Reddy, S. Chinthamreddy, A. Al-Hamdan, Remediation (2001) 85-109.

11. K. Reddy, M. Donahue, R. Saichek, R. Sasaoca, Journal of Air \& Waste Managent Assoc. 99 (1999) 823-830.

12. K. Reddy, U. Parupudi, S. Devulapalli, C. Xu, Journal of Hazardous Materials 55 (1997) 135-158.

13. K. Reddy, A.B. Shirani, Geotechnical and Geological Engineering 15 (1997) 3-29.

14. K. Reddy, S. Chinthamreddy, Waste Management 19 (1999) 269-282.

15. M.V. Vázquez, D.A. Tobón, Afinidad 58 (2001) 437-441.

16. M.V. Vázquez, F. Hernández-Luis, Innovación 15 (2003) 45-51.

17. M.V. Vázquez, F. Hernández-Luis, C.D. Arbelo, D. Grandoso, Portugaliae Electrochim. Acta 22 (2004) 399-410.

18. M.V. Vázquez, F. Hernández-Luis, C.D. Arbelo, D. Grandoso, M. Lemus, Remediation 14 (2004) 105-112.

19. M.V. Vázquez, F. Hernández-Luis, C. D. Arbelo, M. Lemus, Portugaliae Electrochim. Acta 22 (2004) 387-398.

20. M.V. Vázquez, D.A. Tobón, F.L. Gutiérrez, Afinidad 59 (2002) 601-604.

21. G.W. Thomas, Exchangeable cations. "Methods of soils analysis" Part 2. Chemical and Microbiological Properties. Agronomy Monograph no. 9 ( $^{\text {nd }}$ Edition) pp 159-165 A.S.A., Inc Publisher. Madison Wisconsin, USA 